\title{
PRODUCCIÓN DE METANO Y DIGESTIBILIDAD DE MEZCLAS KIKUYO (Pennisetum clandestinum) - PAPA (Solanum tuberosum) ${ }^{1}$
}

\author{
Sandra Lucía Posada-Ochoa ${ }^{2}$, John Fredy Ramírez-Agudelo ${ }^{2}$, Ricardo Rosero-Noguera ${ }^{2}$
}

\begin{abstract}
RESUMEN
Producción de metano y digestibilidad de mezclas kikuyo (Pennisetum clandestinum) - papa (Solanum tuberosum). El objetivo del presente trabajo fue evaluar el efecto de diferentes proporciones kikuyo (Pennisetum clandestinum) - papa (Solanum tuberosum) sobre la producción in vitro de metano. Durante el segundo semestre del año 2012 en la Sede de Investigación Universitaria (Medellín, Colombia), se evaluaron diferentes proporciones kikuyo $(\mathrm{K})$ - papa $(\mathrm{P})$ K/P de 100/0 (T1), 75/25 (T2) y 50/50\% (T3). Los resultados obtenidos fueron procesados a través de análisis de medidas repetidas en el tiempo y de regresión no lineal. Al finalizar el período de incubación (entre 0 y 48 horas), la producción acumulada de gas $(\mathrm{ml} / \mathrm{g} \mathrm{MS}$ ), de metano $(\mathrm{ml} / \mathrm{g} \mathrm{MS}$ ) y la digestibilidad de la materia seca $(\%)$ presentaron diferencias estadísticas significativas $(\mathrm{p}<0,05)$ entre todos los tratamientos, aumentando con mayores niveles de inclusión de papa en la mezcla. En el mismo intervalo de tiempo, la relación entre la digestibilidad de la materia seca y la producción acumulada de gas y de metano $(\mathrm{mg} / \mathrm{ml})$ no mostró diferencia estadísticamente significativa $(\mathrm{p}>0,05)$ entre todos los tratamientos evaluados. El volumen final de gas y de metano $\left(V_{f}\right)$ y su tasa de producción durante todo el proceso fermentativo $(k)$, estimados a partir del modelo Gompertz, también fueron mayores $(\mathrm{p}<0,05)$ para los tratamientos incluyendo papa. La mayor producción de metano es resultado de la superior digestibilidad de la materia seca conforme se incrementó el nivel de carbohidratos no estructurales (CNE) en la mezcla. Bajo el modelo in vitro y a diferencia de los reportes de la literatura, la mayor inclusión de CNE no redujo la producción de metano.
\end{abstract}

Palabras clave: carbohidratos, metanogénesis, técnica in vitro de producción de gases.

\begin{abstract}
Methane production and digestibility of kikuyu grass (Pennisetum clandestinum) and potato (Solanum tuberosum) mixtures. The objective of this study was to evaluate the effect of the mixture of different ratios of kikuyu grass (Pennisetum clandestinum) $(\mathrm{K})$ - potato (Solanum tuberosum) $(\mathrm{P})$ on methane production in vitro, during the second semester of 2012 in the University Research Center (Medellín, Colombia). The treatments consisted of mixtures $\mathrm{K} / \mathrm{P}$ in ratios of 100/0 (T1), 75/25 (T2) and 50/50\% (T3). The results were processed through a repeated measures analysis over time and nonlinear regression. At the end of incubation period (between 0 and 48 hours), the cumulative gas production $\left(\mathrm{ml} / \mathrm{g} \mathrm{MS}_{\mathrm{i}}\right)$, the cumulative methane production $\left(\mathrm{ml} / \mathrm{g} \mathrm{MS}_{\mathrm{i}}\right)$ and the dry matter digestibility were statistically differents $(\mathrm{p}<0.05)$ among all treatments, increasing with higher levels of potato in the mixture. In the same time interval, the relationship between dry matter digestibility and cumulative production of gas and methane $(\mathrm{mg} / \mathrm{ml})$ was statistically equivalent ( $>0.05)$ among all treatments. The final volume of gas and methane $(V f)$ and its rate of production throughout the fermentation process $(k)$, estimated from the Gompertz model, were also higher $(\mathrm{p}<0.05)$ for treatments including potato. Increased methane production is the result of higher dry matter digestibility according CNE level increases in the mixture. Under the in vitro model and in contrast to literature reports, the greater inclusion of CNE did not reduce methane production.
\end{abstract}

Keywords: non-structural carbohydrates, methanogenesis, in vitro gas production technique.

\footnotetext{
Recibido: 4 de febrero, 2013. Aceptado: 18 de marzo, 2014. Parte del proyecto de investigación "Efecto de la edad de cosecha, la suplementación con grano y la utilización de estatinas sobre la producción in vitro de metano de dietas basadas en pasto kikuyo (Pennisetum clandestinum)", formulado como parte de los estudios de posgrado en Ciencias Animales de la Universidad de Antioquia, Medellín, Colombia

2 Universidad de Antioquia, Facultad de Ciencias Agrarias, Grupo de Investigación en Ciencias Agrarias-GRICA. Carrera 75 \# 65-87, AA 1226, Medellín,Colombia.slposada@gmail.com (autor para correspondencia), framirez161@yahoo.com, ricnoguera@gmail.com
} 


\section{INTRODUCCIÓN}

El metano $\left(\mathrm{CH}_{4}\right)$ es producido por los microorganismos ruminales durante la fermentación anaeróbica de los carbohidratos solubles y estructurales consumidos por rumiantes (Kurihara et al., 1999). La importancia de su producción radica en viabilizar el funcionamiento del rumen al disminuir la presión de hidrógeno (Blaut, 1994). No obstante, en la atmósfera este gas tiene la capacidad de almacenar calor veinte veces superior a la del dióxido de carbono $\left(\mathrm{CO}_{2}\right)$, por lo cual los rumiantes se han relacionado con la contribución de gases de efecto invernadero (Arcuri et al., 2006).

Otro efecto adverso de las emisiones de metano se refiere a la pérdida energética que experimentan los animales, lo cual afecta adversamente su desempeño productivo. La producción de este gas representa, en promedio, $8 \%$ de la energía bruta consumida (Resende et al., 2006), valor que puede fluctuar entre el 5,5 y 13\% (Johnson y Ward, 1996; Wolin y Miller, 2006). Por tanto, las emisiones de metano no sólo constituyen un problema ambiental, sino una pérdida energética que juega contra la competitividad del sector ganadero.

El creciente interés mundial por reducir el calentamiento global y direccionar la energía consumida hacia la producción de leche y carne, conlleva a formular estrategias de alimentación tendientes a reducir la emisión de metano en la atmósfera. Con sistemas productivos basados en forraje, la fibra representa un componente dietético que resulta en la producción preferente de acetato, butirato y metano. Sin embargo, el aumento de la fermentación propiónica y la reducción en la relación acetato:propionato, mediante suplementación con carbohidratos no estructurales (CNE), reduce en gran medida la metanogénesis por unidad de alimento ingerido (Johnson y Johnson, 1995; Martin et al., 2010). Doreau et al. (2011) trabajando con ganado de carne, reportaron que la producción de metano, expresada en gramos por día o por kilogramo de materia seca consumido, descendió con incrementos en la proporción de almidón en la dieta.

En algunas poblaciones de los andes colombianos es común cultivar papa (Solanum tuberosum) en fincas lecheras con la finalidad de renovar los potreros. En las épocas del año en que se colecta la cosecha, los precios del tubérculo descienden y cantidades considerables se pierden por la menor utilidad económica derivada de su comercialización. Igualmente, existe una porción de la papa cosechada que por su tamaño reducido y daño físico no puede ser comercializada. En Colombia, tanto los excedentes como los desechos de la papa se han utilizado en la alimentación de vacas lactantes debido a su significativo aporte de CNE y energía (Montoya et al., 2003; Montoya et al., 2004; Betancourt et al., 2012).

Debido a que los estudios in vivo de la metanogénesis consumen tiempo, son costosos y demandan grandes instalaciones y recursos, existe interés creciente en el empleo de técnicas de fermentación in vitro (Navarro-Villa et al., 2011). El objetivo del presente trabajo fue evaluar el efecto de diferentes proporciones kikuyo (Pennisetum clandestium) - papa (Solanum tuberosum) sobre la producción in vitro de metano.

\section{MATERIALES Y MÉTODOS}

\section{Localización}

El experimento se desarrolló durante el segundo semestre del año 2012 en el Laboratorio de Investigación en Nutrición y Alimentación Animal (NUTRILAB). Este laboratorio se encuentra localizado en la Sede de Investigación Universitaria (SIU) de la Universidad de Antioquia, Medellín, Colombia, a $06^{\circ} 13$ ' $55^{\prime \prime} \mathrm{N}$ y $75^{\circ} 34^{\prime} 05^{\prime \prime} \mathrm{O}, 1540 \mathrm{msnm}$ y presenta una temperatura promedio anual de $24^{\circ} \mathrm{C}$, humedad relativa del $68 \%$ y precipitación media anual de 1600 mm (IDEAM, 1999).

\section{Tratamientos experimentales}

Los sustratos evaluados fueron pasto kikuyo (Pennisetum clandestinum) (K) de 45 días de edad y tubérculo de papa sin la epidermis (cutícula o piel) (Solanum tuberosum) $(\mathrm{P})$ para garantizar una mayor concentración de CNE. La descripción química de los sustratos se presenta en el Cuadro 1. Estos alimentos se mezclaron en tres diferentes proporciones para dar lugar a los tratamientos experimentales, a saber: Tratamiento 1 (T1): $100 \% \mathrm{~K}+0 \% \mathrm{P}$; Tratamiento 2 (T2): $75 \% \mathrm{~K}+25 \%$ P; Tratamiento 3 (T3): $50 \% \mathrm{~K}+50 \%$ P. Los alimentos en estado fresco fueron presecados en estufa de ventilación forzada a $65^{\circ} \mathrm{C}$ por 72 horas, 
Cuadro 1. Descripción química del pasto kikuyo (P. clandestinum) y de la papa (S. tuberosum), expresada como porcentaje de la materia seca. Medellín, Antioquia, Colombia. 2012 .

\begin{tabular}{ccc}
\hline Composición química* & Kikuyo $($ P. clandestinum $)$ & Papa $($ S. tuberosum $)$ \\
\hline PB & 19,42 & 9,50 \\
EE & 3,22 & 0,41 \\
MI & 10,03 & 4,83 \\
FDN & 59,87 & 5,20 \\
CNE & 7,46 & 80,0 \\
\hline
\end{tabular}

\footnotetext{
* $\mathrm{PB}=$ proteína bruta, $\mathrm{EE}=$ extracto etéreo, $\mathrm{MI}=$ material inorgánico, $\mathrm{FDN}=$ fibra en detergente
} neutro, $\mathrm{CNE}=$ carbohidratos no estructurales.

y posteriormente, fueron molidos a $1 \mathrm{~mm}$, utilizando molino estacionario Thomas-Wiley modelo 4 (Arthur H. Thomas Company, Philadelphia). Cada tratamiento contó con tres repeticiones, correspondientes a tres diferentes fuentes de inóculo.

\section{Producción de gas y digestibilidad in vitro}

La incubación se realizó en frascos de vidrio con capacidad de $100 \mathrm{ml}$ cada uno, de los cuales se le agregó $0,5 \mathrm{~g}$ de sustrato (K-P) y $45 \mathrm{ml}$ de saliva artificial, preparada de acuerdo con las recomendaciones de McDougall (1948). Los reactivos fueron disueltos en agua destilada y la solución saturada con $\mathrm{CO}_{2}$. Los frascos con la saliva artificial permanecieron en estufa de ventilación forzada a $39^{\circ} \mathrm{C}$ por cuatro horas, antes de la inoculación con fluido ruminal.

El líquido ruminal fue obtenido de tres vacas Holstein Friesian con fístula, que se encontraban consumiendo pasto kikuyo (Pennisetum clandestinum), sal mineralizada y agua a voluntad. La extracción del inóculo se realizó a las 6:00 horas, posterior al consumo de alimento. Antes de la inoculación, el contenido extraído del rumen fue filtrado en paños de algodón, la parte sólida se transfirió a una licuadora con cierta cantidad de líquido ruminal y se licuó por 20 segundos para desprender los microorganismos adheridos a la fibra. Posteriormente, el material licuado fue nuevamente filtrado, saturado con $\mathrm{CO}_{2}$ y conservado en baño maría a $39^{\circ} \mathrm{C}$.

Los frascos de incubación se inocularon con $5 \mathrm{ml}$ de líquido ruminal y posteriormente se sellaron con tapas de caucho. Para su ubicación en la estufa de ventilación forzada a $39^{\circ} \mathrm{C}$ se dispusieron en cajas de icopor que ayudaron a conservar la temperatura. En total fueron incubados 96 frascos, 72 conteniendo sustrato e inóculo (3 tratamientos $* 3$ repeticiones/tratamiento * 4 horarios de lectura $* 2$ réplicas por horario) y 24 correspondientes a los blancos (4 horarios * 3 inóculos * 2 blancos por horario) (frascos con solución tampón e inóculo pero sin sustrato), cuya función fue corregir la producción de gas generada por los microorganismos.

La presión generada por los gases de fermentación, medida en libras por pulgada cuadrada (PSI), fue cuantificada con un transductor digital de presión (OMEGA Modelo PX 605-030GI) a las 6, 12, 24 y 48 horas de incubación, como lo descrito por Theodorou et al. (1994). Para transformar los datos de presión (PSI) $(x)$ en volumen de gas $(\mathrm{ml})(y)$, se utilizó la ecuación $\mathrm{Y}=-0,1375+\left(5,1385^{*} \mathrm{X}\right)+\left(0,0777 * \mathrm{X}^{2}\right)$ obtenida por Posada et al. (2006) y la producción de gas fue expresada por gramo de materia seca incubada $\left(\mathrm{MS}_{\mathrm{i}}\right)$.

La concentración de metano de los tratamientos experimentales fue determinada en el gas acumulado entre los intervalos de lectura 0-6, 6-12, 12-24 y 24-48 horas. La colecta de gas se realizó utilizando una válvula de tres salidas. La primera salida fue conectada a una aguja $(0,6 \mathrm{~mm})$, la segunda conectada al transductor de presión y la tercera a una jeringa plástica que sirvió para la extracción del volumen de gas. La aguja acoplada a la válvula fue insertada a través de la tapa de caucho para la medición de la presión y posteriormente, los gases acumulados en la parte superior del frasco se retiraron con el uso de la 
jeringa hasta el punto en que la presión registrada en el transductor alcanzó a ser cero. El gas colectado en la jeringa fue trasladado y almacenado en bolsas plásticas herméticamente cerradas, a partir de las cuales se tomaron muestras de $100 \mathrm{ul}$, que fueron inyectadas en un cromatógrafo de gases adaptado con un detector de ionización de llama (FID). Las condiciones cromatográficas fueron adaptadas de Apráez et al. (2012) y correspondieron a una columna apolar de $30 \mathrm{~m}, 0,25 \mathrm{~mm}$ y $0,25 \mu \mathrm{m}$, temperatura del puerto de inyección $200^{\circ} \mathrm{C}$, modo de inyección split 50:1, temperatura del detector $250^{\circ} \mathrm{C}$, temperatura del horno $30^{\circ} \mathrm{C}(5 \mathrm{~min})$ hasta completar $200^{\circ} \mathrm{C}\left(30^{\circ} \mathrm{C} / \mathrm{min}\right)$ y helio como gas de arrastre $(1,0 \mathrm{ml} / \mathrm{min})$. Los datos de concentración de metano fueron procesados de acuerdo con la descripción dada por López y Newbold (2007). La producción de este (ml) fue obtenida del producto entre el volumen total de gas $(\mathrm{ml})$ y la concentración relativa de metano. La tasa de producción de gas y de metano $(\mathrm{ml} / \mathrm{g} \mathrm{MSi} / \mathrm{h})$ se obtuvo dividiendo la producción de gas y de metano $(\mathrm{ml} / \mathrm{g} \mathrm{MS}$ ) entre el tiempo transcurrido (horas) en los diferentes intervalos de lectura.

\section{Digestibilidad de la materia seca y factor de par- tición}

La digestibilidad de la materia seca (DMS), expresada en porcentaje, fue determinada a las $6,12,24$ y $48 \mathrm{~h}$. El contenido de cada frasco de incubación fue vertido en crisoles de filtración, previamente identificados y pesados. La materia seca (MS) no digestible fue determinada por secado del material filtrado a $65^{\circ} \mathrm{C}$ por 48 horas y la MS digestible por la diferencia entre la MS incubada y la no digestible. Entre el tiempo cero (inicio del proceso fermentativo) y cada uno de los horarios en que se determinó la producción de gas y la DMS se calculó el factor de partición, correspondiente a los mg de materia seca digestible (MSD) por cada $\mathrm{ml}$ de gas producido (MSD/Volumen gas, $\mathrm{mg} / \mathrm{ml}$ ). La misma relación se realizó entre la MSD y la producción de metano (MSD/Volumen metano, $\mathrm{mg} / \mathrm{ml})$.

\section{Modelación de la producción de gas y de metano}

La ecuación Gompertz, correspondiente a $V_{t}=V_{f}^{*} \exp \left(-k^{*} \exp \left(-A^{*} t\right)\right)$, fue empleada para modelar la producción de gas y de metano de los tratamientos experimentales, donde $V_{t}=$ volumen de gas y de metano (ml) en el tiempo ( $t$, horas), $V_{f}=$ volumen de gas y de metano correspondiente a la completa digestión del sustrato (asíntota) $(\mathrm{ml}), k=$ tasa constante de producción de gas y de metano (\%/hora) y, $A=$ factor constante de eficiencia microbiana (Noguera et al., 2004). El ajuste de los datos a cada modelo y la estimativa de los parámetros se realizó a través del proceso iterativo del algoritmo Marquardt del procedimiento para modelos no lineales PROC NLIN de SAS Institute (2001).

\section{Análisis estadístico de la información}

Para determinar el efecto de los tratamientos sobre las variables respuesta, se realizó un análisis de medidas repetidas en el tiempo con ayuda del procedimiento PROC MIXED de SAS Institute (2001), donde los efectos fijos correspondieron al tratamiento y el tiempo (horarios), y el efecto aleatorio a la fuente de inóculo ruminal (animal). Para comparar el efecto del tratamiento sobre los parámetros estimados por el modelo Gompertz se empleó el procedimiento para modelos lineales PROC GLM de SAS Institute (2001). La prueba de Tukey ajustada a un nivel de significancia del 5\% fue empleada para realizar la comparación de medias.

\section{RESULTADOS Y DISCUSIÓN}

La producción acumulada de gas y de metano fue estadísticamente diferente entre todos los tratamientos $(\mathrm{p}<0,05)$ en los intervalos $0-24$ y $0-48$ horas, presentando valores decrecientes con reducción en la participación de papa en el sustrato incubado (Cuadro 2). Los carbohidratos constituyen el $80 \%$ de la MS de las dietas para rumiantes y pueden ser clasificados como estructurales y no estructurales; los últimos que están constituidos por almidón, azúcares, pectina y $\beta$ glucanos (Van Soest et al., 1994). Chai et al. (2004) al evaluar diferentes fuentes de almidón a través de la técnica de producción de gas concluyeron que el volumen de este producido, es proporcional a la concentración de CNE presentes en la muestra. En este estudio, la participación porcentual de CNE en los tratamientos $\mathrm{T} 1, \mathrm{~T} 2$ y T3 fue 7,$46 ; 25,50$ y $43,73 \%$, respectivamente. El metabolismo microbiano se regula por la canti- 
Cuadro 2. Producción acumulada de gas y de metano, digestibilidad de la materia seca y relación entre la materia seca digestible y el volumen de gas y metano producido en diferentes proporciones kikuyo (P. clandestinum)-papa (S. tuberosum). Medellín, Antioquia, Colombia. 2012.

\begin{tabular}{|c|c|c|c|c|c|c|c|}
\hline \multirow[t]{2}{*}{ Variable } & \multirow[t]{2}{*}{$\begin{array}{c}\text { Intervalo } \\
\text { (horas) }\end{array}$} & \multicolumn{2}{|c|}{$\begin{array}{c}\text { T1 } \\
(\mathrm{K} / \mathrm{P}-100 / 0)\end{array}$} & \multicolumn{2}{|c|}{$\begin{array}{c}\text { T2 } \\
(\mathrm{K} / \mathrm{P}-75 / 25)\end{array}$} & \multicolumn{2}{|c|}{$\begin{array}{c}\text { T3 } \\
(\mathrm{K} / \mathrm{P}-50 / 50)\end{array}$} \\
\hline & & Prom. & EE & Prom. & EE & Prom. & EE \\
\hline \multirow[t]{4}{*}{ PGA (ml/g MS $)$} & $0-6$ & $39,21 \mathrm{a}$ & 1,1256 & $33,57 \mathrm{ab}$ & 2,1283 & $26,25 \mathrm{~b}$ & 1,0807 \\
\hline & $0-12$ & $101,39 \mathrm{~b}$ & 1,8328 & $111,00 \mathrm{a}$ & 5,1845 & $111,13 \mathrm{a}$ & 4,9687 \\
\hline & $0-24$ & $180,19 \mathrm{c}$ & 1,5253 & $233,21 \mathrm{~b}$ & 4,9567 & $270,35 \mathrm{a}$ & 4,7912 \\
\hline & $0-48$ & $254,46 \mathrm{c}$ & 1,6614 & $310,37 \mathrm{~b}$ & 4,9729 & $360,17 \mathrm{a}$ & 1,5011 \\
\hline \multirow[t]{4}{*}{ PMA (ml/g MS $\left.{ }_{i}\right)$} & $0-6$ & 4,35 & 0,2695 & 4,48 & 0,2746 & 3,98 & 0,1784 \\
\hline & $0-12$ & $14,63 \mathrm{~b}$ & 0,8602 & $23,50 \mathrm{a}$ & 1,5379 & $25,71 \mathrm{a}$ & 1,2828 \\
\hline & $0-24$ & $28,55 \mathrm{c}$ & 1,6238 & $45,87 \mathrm{~b}$ & 1,0166 & $55,23 \mathrm{a}$ & 3,0500 \\
\hline & $0-48$ & $43,62 \mathrm{c}$ & 2,8414 & $58,69 \mathrm{~b}$ & 3,0800 & $73,44 \mathrm{a}$ & 3,0603 \\
\hline \multirow[t]{4}{*}{ DMS (\%) } & $0-6$ & $23,16 \mathrm{~b}$ & 1,0383 & 30,98 a & 1,7683 & $22,31 \mathrm{~b}$ & 1,0031 \\
\hline & $0-12$ & $33,99 \mathrm{~b}$ & 1,9429 & $43,54 \mathrm{a}$ & 0,6250 & $44,97 \mathrm{a}$ & 1,3980 \\
\hline & $0-24$ & $47,01 \mathrm{~b}$ & 2,4294 & $61,94 \mathrm{a}$ & 0,8983 & $66,14 \mathrm{a}$ & 2,3107 \\
\hline & $0-48$ & $60,19 \mathrm{c}$ & 1,0974 & $68,26 \mathrm{~b}$ & 1,0110 & $75,90 \mathrm{a}$ & 0,2030 \\
\hline \multirow[t]{4}{*}{$\mathrm{MSD} / \mathrm{VG}(\mathrm{mg} / \mathrm{ml})$} & $0-6$ & $5,93 \mathrm{~b}$ & 0,2601 & 9,37 a & 0,4950 & $8,50 \mathrm{a}$ & 0,0754 \\
\hline & $0-12$ & 3,37 & 0,2164 & 3,84 & 0,2200 & 4,07 & 0,2689 \\
\hline & $0-24$ & 2,61 & 0,1555 & 2,66 & 0,0929 & 2,45 & 0,0887 \\
\hline & $0-48$ & 2,37 & 0,0578 & 2,20 & 0,0635 & 2,11 & 0,0033 \\
\hline \multirow[t]{4}{*}{ MSD/VM (mg/ml) } & $0-6$ & $54,15 \mathrm{~b}$ & 3,1557 & $74,47 \mathrm{a}$ & 4,3926 & $57,18 \mathrm{ab}$ & 2,6889 \\
\hline & $0-12$ & 25,60 & 1,0141 & 17,59 & 0,7150 & 18,02 & 1,0169 \\
\hline & $0-24$ & 16,61 & 0,9510 & 13,52 & 0,4253 & 12,08 & 0,7823 \\
\hline & $0-48$ & 13,92 & 0,7971 & 11,71 & 0,7686 & 10,37 & 0,3942 \\
\hline
\end{tabular}

$\mathrm{K}=$ pasto kikuyo $($ P. clandestinum $), \mathrm{P}=$ papa $($ S. tuberosum $), \mathrm{PGA}=$ producción de gas acumulada, $\mathrm{PMA}=$ producción de metano acumulada, $\mathrm{DMS}=$ digestibilidad de la materia seca, $\mathrm{MSD}=$ materia seca digestible, $\mathrm{MS}_{\mathrm{i}}=$ materia seca incubada

Prom $=$ promedio, $\mathrm{EE}=$ error estándar.

Medias con diferente letra en la fila presentan diferencia estadística $(\mathrm{p}<0,05)$.

dad y por la tasa de hidrólisis de los CNE, las cuales a su vez dependen del procesamiento del sustrato. En este experimento, la mayor disponibilidad de energía fermentable dada por la inclusión de papa molida en el sustrato incubado explicó el mayor volumen de gas y de metano por gramo de materia seca incubada en los intervalos 0-24 y 0-48 horas.

La DMS fue estadísticamente equivalente $(p>0,05)$ entre los tratamientos T2 y T3 en los períodos
0-12 y 0-24, sin embargo, al completar todo el período de incubación (hasta las 48 horas), todos los tratamientos fueron estadísticamente diferentes $(p<0,05)$, con valores superiores a medida que se aumentó la inclusión de papa en el sustrato incubado (Cuadro 2). En el intervalo 0-48 horas, la producción de gas, al igual que la extensión de la degradación aumentó con la participación de CNE en el tratamiento, lo cual se explica por la mayor disponibilidad energética 
dentro del sustrato incubado. El almidón representó la mayor fuente de carbohidratos para el crecimiento microbiano y una correlación positiva existe entre este crecimiento y el aumento en la digestión de la materia seca. Se ha observado que la digestibilidad de la materia seca y orgánica aumentó conforme se incrementó el nivel de carbohidratos fermentables en el rumen (Nussio et al., 2006; Krause y Combs, 2003). De igual manera, Feng et al. (1993) encontraron que un incremento del 29 al 39\% de CNE en raciones para vacas, mejoró sustancialmente la degradación de la materia seca y orgánica en $19,34 \%$ y $11,66 \%$, respectivamente. La extensión de la fermentación de la dieta total en el rumen es dependiente de la cantidad de carbohidratos rápidamente hidrolizables como azúcares, pectinas y almidones (Cone et al., 1989). Pero, la relación negativa entre la DMS y el contenido de carbohidratos estructurales ha sido descrita en la literatura. Mahyuddin (2008) encontraron una correlación negativa entre el contenido de fibra detergente neutra y la digestibilidad in vitro de la materia seca (DIVMS) de forrajes tropicales. También Firdous y Gilani (2002) corroboran la correlación negativa entre los componentes de la pared celular (FDN, FDA, celulosa, hemicelulosa y lignina) y la DIVMS de la planta de maíz entera. Tomando en consideración la composición química de los sustratos incubados (Cuadro 1), los tratamientos T1, T2 y T3 presentaron una relación FDN/CNE correspondiente a $8,03 / 1,1,80 / 1$ y $0,74 / 1$, lo cual igualmente explica la respuesta observada en la DMS.

La extensión o la tasa de digestión de un ingrediente puede ser inhibida o aumentada por la presencia de constituyentes de otro alimento, lo cual ha sido definido como efecto asociativo (Dryden, 2008). En este trabajo, la inclusión de fuentes de almidón como la papa aumentó la digestibilidad total del sustrato incubado, no sólo por su mayor degradabilidad, sino por el efecto asociativo positivo que se origina en la mezcla con alimentos de menor disponibilidad energética. De acuerdo con Nussio et al. (2006), la digestión del almidón es variable por el tipo de fuente utilizada, pero los valores fluctúan entre 65 y $90 \%$.

El comportamiento observado en las variables producción de gas y DMS se corresponde con lo indicado por Menke et al. (1979), quienes encontraron que la producción de gas acumulada en 24 horas estuvo correlacionada con la digestibilidad de la materia orgánica (MO) determinada in vivo. Dryden (2008) señaló que el dióxido de carbono y el metano son producidos en cantidades estequiométricas a la cantidad de $\mathrm{MO}$ fermentada por los microorganismos ruminales. Finalmente, López et al. (1998) han reportado significativas correlaciones entre la tasa fraccional de desaparición de la MS in situ y la tasa fraccional de producción de gas. Al igual que en el presente trabajo, donde superiores digestibilidades de la materia seca entre 0 y 48 $\mathrm{h}$ se relacionaron con mayor producción acumulada de gas en el mismo período (Cuadro 2), Noguera et al. (2006) encontraron una relación lineal positiva entre la degradación de la materia seca y la producción de gas durante el proceso fermentativo, indicando que a medida que más sustrato se degrada mayor volumen se produce. No obstante, mayor producción no necesariamente indica mayor eficiencia microbiana en la utilización del sustrato, lo cual da origen al concepto denominado factor de partición, que relaciona el sustrato degradado $(\mathrm{mg})$ y el volumen de gas producido $(\mathrm{ml})$. Sustratos que registren superior degradación de la materia seca por cada unidad de gas generado se asocian con mayor factor de partición y biomasa microbiana, pero con menor producción de metano (Posada y Noguera, 2005).

En el presente trabajo, los factores de partición resultantes de relacionar MSD/volumen gas y MSD/ volumen metano fueron estadísticamente equivalentes entre tratamientos $(p>0,05)$ en todos los intervalos en estudio, con excepción de la diferencia que se presentó en el intervalo 0-6 h. Ello está indicando que bajo condiciones in vitro, la mayor DMS resultante de la inclusión de CNE fue contrarrestada por una superior producción de gas y de metano, lo que desde el punto de vista ambiental concede a los diferentes tratamientos evaluados el mismo poder contaminante. Dietas que sean más sustentables desde el punto de vista ambiental deben propender por mayores factores de partición. Igualmente, se ha reportado que dietas con mayor valor en esta variable impactan positivamente el consumo de materia seca (Posada y Noguera, 2005), lo cual las hace más competitivas nutricionalmente.

En los intervalos 6-12 y 12-24 horas, la tasa de producción de gas $\left(\mathrm{ml} / \mathrm{h} / \mathrm{g} \mathrm{MS}_{\mathrm{i}}\right)$ presentó diferencia estadística entre todos los tratamientos $(\mathrm{p}<0,05)$, siendo superior para $\mathrm{T} 3$, intermedia para $\mathrm{T} 2$ e inferior para $\mathrm{T} 1$, en tanto que durante el período inicial $(0-6$ horas), T1 registró mayor valor $(\mathrm{p}<0,05)$ respecto los tratamientos que incluyeron papa. Entre 6-12 y 
12-24 horas de incubación, la tasa de producción de metano $(\mathrm{ml} / \mathrm{h} / \mathrm{g} \mathrm{MS}$ ) fue mayor para los tratamientos incluyendo papa (T2 y T3) respecto $\mathrm{T} 1$, con el cual presentaron diferencia estadísticamente significativa $(\mathrm{p}<0,05)$ (Cuadro 3). La ausencia de diferencia estadística entre tratamientos en estas variables para el intervalo $24-48 \mathrm{~h}$ puede deberse a la completa digestión de los CNE de la papa. Chai et al. (2004) encontraron que la mayor degradación del almidón ocurrió después de las 6 horas de incubación y fue completada dentro de las primeras 24 horas.

En el Cuadro 4 se presentan los parámetros estimados por el modelo Gompertz. Se observa que el volumen final de gas y de metano $\left(V_{f}\right)$ y su tasa de producción durante todo el proceso fermentativo $(k)$ fueron mayores $(\mathrm{p}<0,05)$ para los tratamientos incluyendo papa. La superioridad en estos parámetros se corresponde con la mayor producción acumulada de gas y de metano (Cuadro 2) y con su mayor tasa de producción (Cuadro 3). Noguera et al. (2006) evaluando el efecto de niveles crecientes de papa $(0,15,30,45$ y $60 \%$ ) en la cinética de fermentación in vitro del pasto kikuyo, encontraron mayor volumen final de gas y tasa de producción de gas conforme los niveles de papa se incrementaron, lo que se corresponde con la tendencia observada en el presente experimento. El factor de eficiencia microbiana sólo presentó diferencia estadística significativa $(\mathrm{p}<0,05)$ entre $\mathrm{T} 2$ y $\mathrm{T} 3$ respecto $\mathrm{T} 1$ cuando se modelaron los datos de producción de gas.

La eficiencia de crecimiento microbiano puede ser expresada en términos de materia seca de células microbianas producidas por mol de sustrato fermentado $\left(\mathrm{Y}_{\mathrm{SUB}}\right)$ o mol de ATP disponible $\left(\mathrm{Y}_{\mathrm{ATP}}\right)$ (Valadares y Santos, 2006). Según Van Soest (1994), la eficiencia microbiana es definida como la proporción de energía del sustrato fijado dentro de las células. La superioridad del parámetro A en los tratamientos incluyendo papa se explica por la mayor presencia de CNE y la mayor digestibilidad del sustrato incubado.

La cantidad de metano que se produce, necesaria para consumir hidrógeno, está relacionada con los productos finales de la fermentación de los carbohidratos. Una relación inversa existe entre la producción de propionato y de metano, toda vez que las moléculas a partir de las cuales se origina este gas, dióxido de carbono e hidrógeno, son subproductos de la conversión de glucosa en acetato y butirato. Raciones abundantes en cereales presentan un coeficiente molar acetato:propionato igual a uno (1), en tanto raciones altas en forraje presentan un coeficiente molar igual a tres (3) (Nussio et al., 2006). Desde este punto de vista, los tratamientos 2 y 3 deberían de haber registrado

Cuadro 3. Tasa de producción de gas y de metano de diferentes proporciones kikuyo (P. clandestinum)-papa (S. tuberosum) en diferentes intervalos de tiempo. Medellín, Antioquia, Colombia. 2012.

\begin{tabular}{|c|c|c|c|c|c|c|c|}
\hline \multirow[t]{2}{*}{ Variable } & \multirow[t]{2}{*}{$\begin{array}{c}\text { Intervalo } \\
\text { (horas) }\end{array}$} & \multicolumn{2}{|c|}{$\begin{array}{c}\text { T1 } \\
(\mathrm{K} / \mathrm{P}-100 / 0)\end{array}$} & \multicolumn{2}{|c|}{$\begin{array}{c}\text { T2 } \\
(\mathrm{K} / \mathrm{P}-\mathbf{7 5} / 25)\end{array}$} & \multicolumn{2}{|c|}{$\begin{array}{c}\text { T3 } \\
(\mathbf{K} / \mathbf{P}-50 / 50)\end{array}$} \\
\hline & & Prom. & $\mathbf{E E}$ & Prom. & $\mathbf{E E}$ & Prom. & $\mathbf{E E}$ \\
\hline \multirow[t]{4}{*}{ TPG (ml/h/g MS $\left.{ }_{\mathrm{i}}\right)$} & $0-6$ & $6,54 \mathrm{a}$ & 0,1867 & $5,59 \mathrm{~b}$ & 0,3527 & $4,37 \mathrm{c}$ & 0,1826 \\
\hline & 6-12 & $10,36 \mathrm{c}$ & 0,1538 & $12,91 \mathrm{~b}$ & 0,5174 & $14,15 \mathrm{a}$ & 0,6501 \\
\hline & $12-24$ & $6,57 \mathrm{c}$ & 0,0800 & $10,19 \mathrm{~b}$ & 0,1287 & $13,27 \mathrm{a}$ & 0,0656 \\
\hline & $24-48$ & 3,16 & 0,0233 & 3,21 & 0,0835 & 3,74 & 0,2223 \\
\hline \multirow[t]{4}{*}{$\left.\mathrm{TPM}(\mathrm{ml} / \mathrm{h} / \mathrm{g} \mathrm{MS})_{\mathrm{i}}\right)$} & $0-6$ & 0,72 & 0,0421 & 0,75 & 0,0414 & 0,66 & 0,0384 \\
\hline & $6-12$ & $1,71 \mathrm{~b}$ & 0,1115 & $3,17 \mathrm{a}$ & 0,1262 & $3,62 \mathrm{a}$ & 0,1702 \\
\hline & $12-24$ & $1,16 \mathrm{~b}$ & 0,0231 & $1,86 \mathrm{a}$ & 0,0433 & $2,46 \mathrm{a}$ & 0,0384 \\
\hline & 24-48 & 0,63 & 0,0264 & 0,54 & 0,0253 & 0,76 & 0,0461 \\
\hline
\end{tabular}

$\mathrm{K}=$ pasto kikuyo $($ P. clandestinum $), \mathrm{P}=$ papa $(S$. tuberosum $) . \mathrm{TPG}=$ tasa de producción de gas, $\mathrm{TPM}=$ tasa de producción de metano, $\mathrm{MS}_{\mathrm{i}}=$ materia seca incubada.

Prom $=$ promedio, $\mathrm{EE}=$ error estándar.

Medias con diferente letra en la fila presentan diferencia estadística significativa $(p<0,05)$. 
Cuadro 4. Parámetros estimados por el modelo Gompertz para la producción de gas y de metano de diferentes proporciones kikuyo (P. clandestinum) - papa (S. tuberosum). Medellín, Antioquia, Colombia. 2012.

\begin{tabular}{|c|c|c|c|c|c|c|}
\hline \multirow[t]{3}{*}{ Tratamiento } & \multicolumn{6}{|c|}{ Parámetros estimados ${ }^{1}$} \\
\hline & \multicolumn{3}{|c|}{ Gas } & \multicolumn{3}{|c|}{ Metano } \\
\hline & $V_{f}$ & $k$ & $A$ & $V_{f}$ & $k$ & $A$ \\
\hline $\mathrm{T} 1(\mathrm{~K} / \mathrm{P}-100 / 0)$ & $\begin{array}{l}264,23 \mathrm{c} \\
(1,7609)\end{array}$ & $\begin{array}{l}3,0006 \mathrm{c} \\
(0,0395)\end{array}$ & $\begin{array}{c}0,0885 \mathrm{~b} \\
(0,0002)\end{array}$ & $\begin{array}{l}46,18 \mathrm{~b} \\
(4,2758)\end{array}$ & $\begin{array}{l}3,5340 \mathrm{~b} \\
(0,1773)\end{array}$ & $\begin{array}{c}0,0925 \\
(0,0036)\end{array}$ \\
\hline $\mathrm{T} 2(\mathrm{~K} / \mathrm{P}-75 / 25)$ & $\begin{array}{l}315,57 \mathrm{~b} \\
(4,9282)\end{array}$ & $\begin{array}{l}4,1120 \mathrm{~b} \\
(0,0845)\end{array}$ & $\begin{array}{l}0,1106 \mathrm{a} \\
(0,0013)\end{array}$ & $\begin{array}{c}58,86 \mathrm{a} \\
(3,6837)\end{array}$ & $\begin{array}{l}5,8050 \mathrm{a} \\
(1,5407)\end{array}$ & $\begin{array}{c}0,1439 \\
(0,0033)\end{array}$ \\
\hline T3 (K/P-50/50) & $\begin{array}{l}365,67 \text { a } \\
(2,1850)\end{array}$ & $\begin{array}{l}5,1015 \mathrm{a} \\
(0,0586)\end{array}$ & $\begin{array}{c}0,1190 \mathrm{a} \\
(0,0033)\end{array}$ & $\begin{array}{c}73,69 a \\
(2,6042)\end{array}$ & $\begin{array}{l}5,1540 \mathrm{a} \\
(0,0997)\end{array}$ & $\begin{array}{c}0,1248 \\
(0,0053)\end{array}$ \\
\hline
\end{tabular}

${ }^{1}$ Valores correspondientes a la media ( \pm error estándar).

$V_{f}=$ volumen de gas y de metano correspondiente a la completa digestión del sustrato (asíntota) (ml); $\mathrm{k}=$ tasa constante de producción de gas (\%hora-1); $A$ = factor constante de eficiencia microbiana.

$\mathrm{K}=$ pasto kikuyo $(P$. clandestinum), $\mathrm{P}=$ papa $(S$. tuberosum $)$

Medias con diferente letra en la columna presentan diferencia estadística significativa $(\mathrm{p}<0,05)$.

menor producción de metano, lo cual no fue evidenciado en el presente experimento (Cuadro 2, 3 y 4). La mayor producción de metano en estos tratamientos, respecto el $\mathrm{T} 1$, posiblemente se explique por su mayor digestibilidad sin que se afecten las condiciones de $\mathrm{pH}$ al interior del frasco de fermentación.

Navarro-Villa et al. (2011) indican que existe preocupación acerca de la conveniencia de la técnica in vitro de producción de gases para evaluar la metanogénesis en alimentos de diferente composición química y degradabilidad, cuyos resultados contrastan con los informes in vivo, en los cuales dietas basadas en granos de cereales registran menor producción de metano. In vitro, la capacidad de amortiguación del medio impide la disminución del pH que normalmente se produce en el rumen cuando se ofrecen alimentos rápidamente fermentables. In vivo, las bacterias metanogénicas son sensibles a los cambios en la ración de los animales. Aumentos en la tasa de pasaje de la digesta y en su tasa de fermentación, así como reducción de la rumia o del $\mathrm{pH}$ son factores que conducen a la reducción de metanógenos y de la cantidad de hidrógeno disponible para la formación de metano (Nussio et al., 2006). Además, la solución fuertemente tamponada del modelo in vitro no produce el bajo $\mathrm{pH}$ que se asocia con la inhibición de bacterias fibrolíticas, lo cual no reduce la relación acetato:propionato que, como ya se indicó, afecta la disponibilidad de hidrógeno para la producción de metano (Navarro-Villa et al., 2011).

\section{LITERATURA CITADA}

Apráez, J.E., J.M. Delgado, y J.P. Narváez. 2012. Composición nutricional, degradación in vitro y potencial de producción de gas de herbáceas, arbóreas y arbustivas encontradas en el trópico alto de Nariño. Livestock Research for Rural Development 24 (44). http://www. lrrd.org/lrrd24/3/apra24044.htm (Consultado 20 ene. 2013).

Arcuri, P.B., F.C.Ferraz, y J.C. Carneiro. 2006. Microbiologia do rúmen. En: T.T. Berchielli, A.V. Pires, y S.G. Oliveira, editores, Nutrição de ruminantes. FUNEP, Sao Paulo, Brasil. p. 111-150.

Betancourt, J.E., H.B. Cuastumal, S.P. Rodríguez, J.F. Navia, y E.G. Insuasty. 2012. Alimentación de vacas holstein con suplemento de papa de desperdicio (Solanum tuberosum) y acacia negra (Acacia decurrens), y su efecto en la calidad de leche. Investig. Pecu. 1(2): 41-51.

Blaut, M. 1994. Metabolism of methanogens. Antonie van Leeuwenhoek 66(1-3):187-208. 
Chai, W.Z., Ah. Van Gelder, y J.W. Cone. 2004. Relationship between gas production and starch degradation in feed samples. Animal Feed Science and Technology 114(1):195-204.

Cone, J.W., W. Cline-Theil, A. Malestein, y A. Klooster. 1989. Degradation of starch by incubation with rumen fluid. A comparison of different starch sources. Journal of the Science of Food and Agriculture 49(2):173-183.

Doreau, M., H.M.G. Van Der, D. Micol, H. Dubroeucq, J. Agabriel, Y. Rochette, y C. Martin. 2011. Enteric methane production and greenhouse gases balance of diets differing in concentrate in the fattening phase of a beef production system. Journal of Animal Science 89(8):2518-2528, doi:10.2527/jas.2010-3140.

Dryden, G. McL. 2008. Animal nutrition science. CABI, Cambridge, UK.

Feng, P., W.H. Hoover, T.K. Miller, y R. Blauwiekel. 1993. Interactions of fiber and nonstructural carbohydrates on lactation and ruminal function. Journal of Dairy Science 76(5):1324-1333.

Firdous, R., y A.H. Gilani. 2002. Prediction of dry matter digestibility of maize (Zea mays) fodder from chemical composition. Pakistan Journal of Agricultural Sciencies 39(1):56-62.

IDEAM (Instituto de Hidrología, Meteorología y Estudios Ambientales). 1999. Cartas climatológicas mensuales, Aeropuerto Olaya Herrera (Medellín). Disponible en http://bart.ideam.gov.co/cliciu/mede/temperatura.htm (Consultado 1 mar. 2013).

Johnson, K.A., y D.E. Johnson. 1995. Methane emissions from cattle. Journal of Animal Science 73(8):24832492.

Johnson, D.E., y G.M. Ward. 1996. Estimates of animal methane emissions. Environmental Monitoring and Assessment 42(1-2):133-141.

Krause, K.M., y D.K. Combs. 2003. Effects of forage particle size, forage source and grain fermentability on performance and ruminal $\mathrm{ph}$ in midlactation cows. Journal of Dairy Science 86(4):1382-1397.

Kurihara, M., T. Magner, R.A. Hunter, y G.J. Mccrabb. 1999. Methane production and energy partition of cattle in the tropics. British Journal of Nutrition 81(3):227-234.

López, S., M.D. Carro, J.S. González, y F.J. Ovejero. 1998. Comparison of different in vitro and in situ methods to estimate the extent and rate of degradation of hays in the rumen. Animal Feed Science and Technology 73(1-2):99-113.
Lopez, S., y C.J. Newbold. 2007. Analysis of methane. En: H.P.S. Makkar, y P.E. Vercoe, editores, Measuring methane production from ruminants. IAEA, FAO, Springer, Dordrecht, The Netherlands. p. 1-13.

Mahyuddin, P. 2008. Relationship between chemical component and in vitro digestibility of tropical grasses. Journal of Biosciences 15(2):85-89.

Martin, C., D.P. Morgavi, y M. Doreau. 2010. Methane mitigation in ruminants: From microbe to the farm scale. Animal 4(3):351-365.

Mc Dougall, E.I. 1948. The composition and output of sheep's saliva. Biochemical Journal 43(1):99-109.

Menke, K.H., L. Raab, A. Salewski, H. Steingass, D. Fritz, y W. Schneider. 1979. The estimation of the digestibility and metabolizable energy content of ruminant feedingstuffs from the gas production when they are incubated with rumen liquor in vitro. Journal Agricultural Science 93(1):217-222.

Montoya, N.F., I.D. Pino, y H.J. Correa. 2003. Efecto de la suplementación con diferentes niveles de papa sobre algunos parámetros productivos y metabólicos en vacas lactantes. Rev. Col. Cienc. Pec. 16(4) (Supl):71.

Montoya, N.F., I.D. Pino, y H.J. Correa. 2004. Evaluación de la suplementación con papa (Solanum tuberosum) durante la lactancia en vacas Holstein. Rev. Col. Cienc. Pec. 17(3):241-249.

Navarro-Villa, A., M. O’brien, S. Lopez, T.M. Boland, y P. O'kiely. 2011. Modifications of a gas production technique for assessing in vitro rumen methane production from feedstuffs. Animal Feed Science and Technology 166-167:163-174.

Noguera, R.R., E.O. Saliba, y R.M. Mauricio. 2004. Comparación de modelos matemáticos para estimar los parámetros de degradación obtenidos a través de la técnica de producción de gas. Livestock Research for Rural Development 16 (11). http://www.lrrd.org/ lrrd16/11/nogu16086.htm (Consultado 15 oct. 2013).

Noguera, R.R., I.C. Ramírez, y D.M. Bolívar. 2006. Efecto de la inclusión de papa (Solanum tuberosum) en la cinética de fermentación in vitro del pasto kikuyo (Pennisetum clandestinum). Livestock Research for Rural Development 18(62). http://www.lrrd.org/ lrrd18/5/nogu18062.htm (Consultado 5 ene. 2013).

Nussio, L.G., F.P. Campos, y M.L. Moreira. 2006. Metabolismo de carboidratos estruturais. En: T.T. Berchielli, A.V. Pires, y S.G. Oliveira, editores, Nutrição de ruminantes. FUNEP, Sao Paulo, Brasil. p. 183-228. 
Posada, S.L., y R.R. Noguera. 2005. Técnica in vitro de producción de gases: Una herramienta para la evaluación de alimentos para rumiantes. Livestock Research for Rural Development 17(36). http://www.lrrd.org/ lrrd17/4/posa17036.htm (Consultado 7 feb. 2013).

Posada, S.L., R.R. Noguera, y D.M. Bolívar. 2006. Relación entre presión y volumen para la implementación de la técnica in vitro de producción de gases en Medellín, Colombia. Revista Colombiana de Ciencias Pecuarias 19(4):407-414.

Resende, K.T., I.A. Almeida, y M.H. Machado, MH. 2006. Metabolismo de energia. En: T.T. Berchielli, A.V. Pires, y S.G. Oliveira, editores, Nutrição de ruminantes. FUNEP, Sao Paulo, Brasil. p. 311-332.

SAS Institute. 2001. SAS/STAT: Guide for personal computer. Versión 8.2. SAS Inst., Cary, NY, USA.
Theodorou, M.K., B.A. Williams, M.S. Dhanoa, A.B. Mcallan, y J. France. 1994. A simple gas production method using a pressure transducer to determine the fermentation kinetics of ruminant feeds. Animal Feed Science and Technology 48(3-4):185-197.

Valadares Filho, S.C., y D. Santos Pina. 2006. Fermentaçao ruminal. En: T.T. Berchielli, A.V. Pires, y S.G. Oliveira, editores, Nutrição de ruminantes. FUNEP, Sao Paulo, Brasil. p. 151-182.

Van Soest, P.J. 1994. Nutritional ecology of the ruminant. $2^{\text {nd }}$ ed. Cornell University Press, New York, USA.

Wolin, M.J., y T.L. Miller. 2006. Control of rumen methanogenesis by inhibiting the growth and activity of methanogens with hydroxymethylglutaryl-SCoA inhibitors. International Congress Series 1293:131137. 